\title{
Immunoglobulins in children with epilepsy: the Dutch Study of Epilepsy in Childhood
}

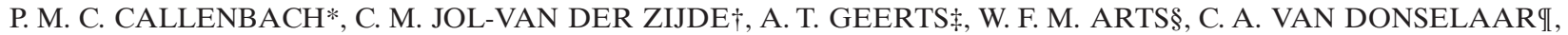 \\ A. C. B. PETERSII, H. STROINK**, O. F. BROUWER*†† \& M. J. D. VAN TOL $\dagger *$ Department of Neurology, Leiden University \\ Medical Centre, Leiden, $\dagger$ Department of Paediatrics, Leiden University Medical Centre, Leiden, $\ddagger$ Department of Neurology, Erasmus \\ Medical Centre Rotterdam, Rotterdam, §Department of Paediatric Neurology, Erasmus Medical Centre Rotterdam and Sophia Children's \\ Hospital, Rotterdam, II Department of Paediatric Neurology, University Medical Centre Utrecht, and Wilhelmina Children's Hospital, \\ Utrecht, **Department of Paediatric Neurology, St. Elisabeth Hospital, Tilburg, and ††Department of Neurology, University Hospital \\ Groningen, Groningen, the Netherlands
}

(Accepted for publication 18 December 2002)

\begin{abstract}
SUMMARY
In an unselected cohort of 282 children, serum immunoglobulin (Ig) concentrations were determined shortly after the first presentation with one or more unprovoked epileptic seizures and before the start of treatment with anti-epileptic drugs (AEDs), and after 9-18 months of AEDs use. At intake, IgA, $\mathrm{IgG} 1, \mathrm{IgG} 2$ and $\mathrm{IgG} 4$ concentrations were significantly higher than published reference values in healthy age-matched controls. In a subset of 127 children, Ig levels at intake were compared with those after AEDs use for 9-18 months. IgA and IgG4 levels had decreased significantly to normal concentrations, but IgG1 and IgG3 levels increased significantly. To determine the influence of AEDs, Ig levels in children who used carbamazepine or valproic acid monotherapy were analysed separately. The use of carbamazepine was associated with a significant decrease of $\operatorname{IgA}$ and $\mathrm{IgG} 4$ levels, and the use of valproic acid with a significant decrease of $\operatorname{IgA}$ and increase of $\mathrm{IgG1}$ levels. In conclusion, humoral immunity is already altered in children shortly after the first presentation with epileptic seizures. Whether this is the consequence of an exogenous event, and to what extent this is related to an interaction of the central nervous system and the immune system, remains to be evaluated. Treatment with AEDs, such as carbamazepine and valproic acid, is associated with significant changes of Ig (sub)class concentrations.
\end{abstract}

Keywords carbamazepine children epilepsy immunoglobulins valproic acid

\section{INTRODUCTION}

Based on the bidirectional interaction between the central nervous system (CNS) and the immune system, it is attractive to speculate that immune mechanisms may be involved in the pathogenesis of at least some forms of epilepsy or that epileptic seizures may affect the immune system indirectly $[1,2]$. The implication of autoimmune mechanisms in the development of epilepsy has been demonstrated most convincingly for Rasmussen's encephalitis, in which antiglutamate receptor 3 antibodies reacting with neuronal antigen probably play a central role in the disease process [3]. Another argument for immunological mechanisms being

Correspondence: M.J.D. van Tol, Department of Paediatrics, Leiden University Medical Centre, PO Box 9600, 2300 RC Leiden, the Netherlands.

E-mail: m.j.d.van_tol@lumc.nl involved is the observation that some patients suffering from this and other forms of intractable epilepsy, i.e. West syndrome, Lennox-Gastaut syndrome and Landau-Kleffner syndrome, may benefit from treatment with intravenous immunoglobulins (IVIg) $[4,5]$. The mechanism of action of IVIg remains, however, currently unknown.

Many reports have been published concerning the effects of the treatment with anti-epileptic drugs (AEDs), e.g. phenytoin [6-9], carbamazepine [10-13] and valproic acid [14-16], on humoral and cellular immunity. From these studies, it became clear that a reversible induction of a selective $\operatorname{IgA}$ deficiency might occur in some patients receiving phenytoin [8], and that carbamazepine medication might be associated with selective reduction of the IgG2 subclass concentration [11]. However, useful interpretation of the results of the majority of the publications is hampered by the lack of data obtained shortly after the onset of seizures and before the start of treatment. Furthermore, the studied populations are relatively small, limited mainly to adult 
patients, or contained a heterogeneous population of adults and children.

We investigated serum immunoglobulins in a large cohort of children from their first presentation with unprovoked epileptic seizures onwards. Here, we present quantitative data of the major immunoglobulin ( $\mathrm{Ig}$ ) isotypes and IgG subclasses in serum samples obtained at intake and after use of AEDs for 9-18 months. The effects of carbamazepine or valproic acid given as monotherapy on Ig serum levels were investigated separately.

\section{METHODS}

\section{Patients}

During an inclusion period of 4 years, 556 children aged 1 month to 16 years presenting in one of the participating hospitals with one or more newly diagnosed unprovoked seizures or at least one status epilepticus were enrolled in the Dutch Study of Epilepsy in Childhood. Detailed inclusion and exclusion criteria and investigations performed are reported elsewhere [1719]. Only if a child had experienced two or more seizures did the paediatric neurologist consider whether to treat the child and select the AEDs. Valproic acid and carbamazepine were used as first-line drugs in all children [17]. The epilepsy was judged not to be controlled adequately if at least two first-line and one second-line drug were tried up to maximum tolerated dosages unsuccessfully.

Three paediatric neurologists involved in the study classified independently the seizures and epilepsy syndromes as idiopathic, remote symptomatic or cryptogenic according to the revised classifications of the International League Against Epilepsy (ILAE) [20,21]. Idiopathic epilepsies are epileptic syndromes with particular clinical characteristics and with specific EEG findings. They are of unknown origin but have a presumed genetic aetiology. Remote symptomatic epilepsies are considered the consequence of a known or suspected disorder of the central nervous system resulting in a static encephalopathy. Cryptogenic epilepsies are epilepsies of unknown origin that do not conform the criteria for the symptomatic or idiopathic categories [22]. All mentally retarded children with epilepsy of unknown aetiology were classified as having remote symptomatic epilepsy. Outcome was assessed with the help of the terminal remission: if the child had been seizure-free for 12 months or more at the end of the follow-up of 5 years, outcome was qualified as 'good', if 6-12 months as 'fair', and if less than 6 months as 'poor' [19].

The study had been approved by the medical ethical committees of all participating hospitals. Parents or caregivers gave their written informed consent.

\section{Immunology}

For several reasons, e.g. protocol deviations by the paediatric neurologist and refusal of the parents, serum samples were not available from 274 of the 556 children who met the inclusion criteria and were enrolled in the Dutch Study of Epilepsy in Childhood. Samples were obtained from the remaining 282 children at intake alone $(n=155)$ or at intake and after the use of AEDs for 918 months $(n=127)$ and stored at $-20^{\circ} \mathrm{C}$. Serum concentrations of $\mathrm{IgM}$ and $\mathrm{IgA}$ were quantified by single radial immunodiffusion (Endoplates, Kallestad, Austin, TX, USA) or nephelometry (BN 100 nephelometer, Behring, Marburg, Germany; antisera from Behring). The concentrations of IgG1, IgG2, IgG3 and IgG4 were measured by dot-immunobinding assay as previously described [23] or nephelometry (BN 100 nephelometer; antisera from the Central Laboratory of the Netherlands Red Cross Blood Transfusion Service (CLB), Amsterdam, the Netherlands).

To validate that the reported median and range of $\mathrm{Ig}$ (sub)class concentrations in serum samples of age-matched controls (reference values for IgM and IgA: Cejka et al. 1974 [24], reference values for the IgG subclasses: CLB, 1997 [25]) could be applied in our experimental setting, serum samples obtained from 76 healthy children were analysed. This control group consisted of sibling donors for recipients of a HLA-identical bone marrow graft that were transplanted at the Department of Paediatrics of the Leiden University Medical Centre.

\section{Statistical analysis}

Normal distribution was tested with the Kolmogorov-Smirnov test. Clinical characteristics were compared between the children from whom a blood sample was taken at intake and the children from whom no blood sample was taken, and between children from whom two blood samples were obtained (at intake and after AEDs use for 9-18 months) and children from whom only a blood sample was obtained at intake using the Pearson $\chi^{2}$ test (sex, aetiology, distribution epilepsy syndromes) and the MannWhitney rank-sum test (age at onset, Ig ratios). Furthermore, differences in clinical characteristics between the distinctive aetiologies were analysed with the Kruskal-Wallis test (age at taking blood sample and outcome) and the Pearson $\chi^{2}$ test (distribution epilepsy syndromes).

Because normal ranges of Ig (sub)classes are age-dependent for each (sub)class, we corrected for age by calculating ratios: the observed Ig (sub)class concentration of each child was divided by the reported median Ig (sub)class concentration in healthy children of the corresponding age group [24,25]. Using the Wilcoxon signed-ranks test, we tested whether the calculated ratios in the cohort of which a blood sample was obtained at intake differed significantly from 1 (if the ratio was 1 , the observed Ig concentration equalled the median concentration of the corresponding age), and whether Ig ratios differed between intake and after AEDs use for 9-18 months. Results were adjusted for multiple testing with the Bonferroni correction. Furthermore, we tested whether children with Ig concentrations outside the normal range of the corresponding age group differed from children with Ig concentrations within the normal range with regard to age, aetiology, distribution of epilepsy syndromes and outcome applying the Pearson $\chi^{2}$ test (aetiology, distribution of epilepsy syndromes) and the Mann-Whitney rank-sum test (age, outcome). We also tested whether the Ig ratios differed between the group with one seizure and the group with multiple seizures (Mann-Whitney rank sum test) or between the different epilepsy syndromes as classified by the ILAE (Kruskal-Wallis test) [21].

\section{RESULTS}

At intake, a blood sample was taken of 282 (51\%; 133 boys, 149 girls) of the 556 children included in the Dutch Study of Epilepsy in Childhood. The median period between the first seizure and obtaining this blood sample was 69 days (range 0 days -6 years). No significant differences were found in sex, aetiology and epilepsy syndrome distribution between the children from whom a blood sample was taken and the children from whom no blood 
sample was taken. Children from whom no blood sample was obtained at intake $(n=274)$ had a significantly lower age at the onset of their epilepsy than children from whom a blood sample was obtained at intake $(n=282$; median age at onset $5 \cdot 1$ and $6 \cdot 1$ years, respectively; $P<0 \cdot 05)$.

Of the 282 children in our cohort, 164 (58\%) suffered from seizures of idiopathic aetiology, 44 (16\%) from seizures of cryptogenic aetiology and $74(26 \%)$ from seizures of remote symptomatic aetiology (Table 1). Twenty-eight children in our cohort experienced a single, unprovoked seizure. Epilepsy syndrome distribution differed significantly between the three groups $(P<0 \cdot 001)$ : children with epilepsy of idiopathic aetiology suffered relatively more often from generalized epilepsy and less from localization-related epilepsy than the other two groups, and children with epilepsy of cryptogenic aetiology suffered relatively less frequently from generalized epilepsy than children with epilepsy of remote symptomatic aetiology $(P<0 \cdot 001$, Table 1$)$. Furthermore, outcome differed significantly between the three groups $(P<0 \cdot 001)$ : children with idiopathic aetiology had a significantly better outcome than both children with cryptogenic aetiology and children with remote symptomatic aetiology $(P<0 \cdot 001$, data not shown).
Immunoglobulin levels at intake

At intake, levels of $\mathrm{IgA}, \mathrm{IgG} 1, \mathrm{IgG} 2$ and $\mathrm{IgG} 4$ were significantly higher in our cohort of 282 children than the reported median Ig (sub)class levels of healthy children of the corresponding ages (the ratios were significantly higher than $1, P<0 \cdot 001$, Fig. 1). Sixty-three children (22\%) had an IgG1 concentration above the upper limit of the normal range for the corresponding age. For the other Ig (sub)classes, up to $6 \%$ of the children had concentrations below the lower limit, and up to $9 \%$ had concentrations above the upper limit of the normal range. The numbers of children with a serum concentration outside the normal range were: for IgM, 16 below the lower limit and 11 above the upper limit; for IgA, 18 and 18; for $\operatorname{IgG} 2,8$ and 26; for $\operatorname{IgG} 3,1$ and 0 ; and for IgG4, 3 and 13, respectively (Fig. 1, grey areas). Two children had an absolute IgA deficiency, and one other child an absolute IgG3 deficiency. Aetiology (i.e. idiopathic, remote symptomatic, cryptogenic), epilepsy syndrome distribution (i.e. localization-related, generalized, single seizure), age at intake and outcome were not significantly different between children with Ig concentrations below the lower limit or above the upper limit of the normal range and those with Ig concentrations within the normal range. This observation holds for all

Table 1. Clinical characteristics of the children from whom a blood sample was taken at intake $(n=282)$

\begin{tabular}{|c|c|c|c|c|}
\hline & Idiopathic & Cryptogenic & Remote symptomatic & Total \\
\hline Number $(\%)$ & $164(58 \cdot 2)$ & $44(15 \cdot 6)$ & $74(26 \cdot 2)$ & $282(100 \cdot 0)$ \\
\hline Median age at taking blood sample (year) & $6 \cdot 5$ & $7 \cdot 1$ & $5 \cdot 4$ & $6 \cdot 3$ \\
\hline \multicolumn{5}{|l|}{ Distribution of epilepsy syndromes ${ }^{\mathrm{a}}$} \\
\hline Localization-related (\%) & $22(13 \cdot 4)^{b}$ & $41(93 \cdot 2)$ & $39(52 \cdot 7)$ & $102(36 \cdot 2)$ \\
\hline Generalized $(\%)$ & $114(69 \cdot 5)$ & $3(6 \cdot 8)$ & $31(41 \cdot 9)$ & $148(52 \cdot 5)$ \\
\hline Other $(\%)$ & $3(1 \cdot 8)$ & & $1(1 \cdot 4)$ & $4(1 \cdot 4)$ \\
\hline Single seizure $(\%)$ & $25(15 \cdot 2)$ & & $3(4 \cdot 1)$ & $28(9 \cdot 9)$ \\
\hline
\end{tabular}

${ }^{a}$ Distribution according to the revised classification of the International League Against Epilepsy, 1989 [21]. ${ }^{b}$ Epilepsy syndrome distribution differed significantly between groups $(P<0 \cdot 001)$.

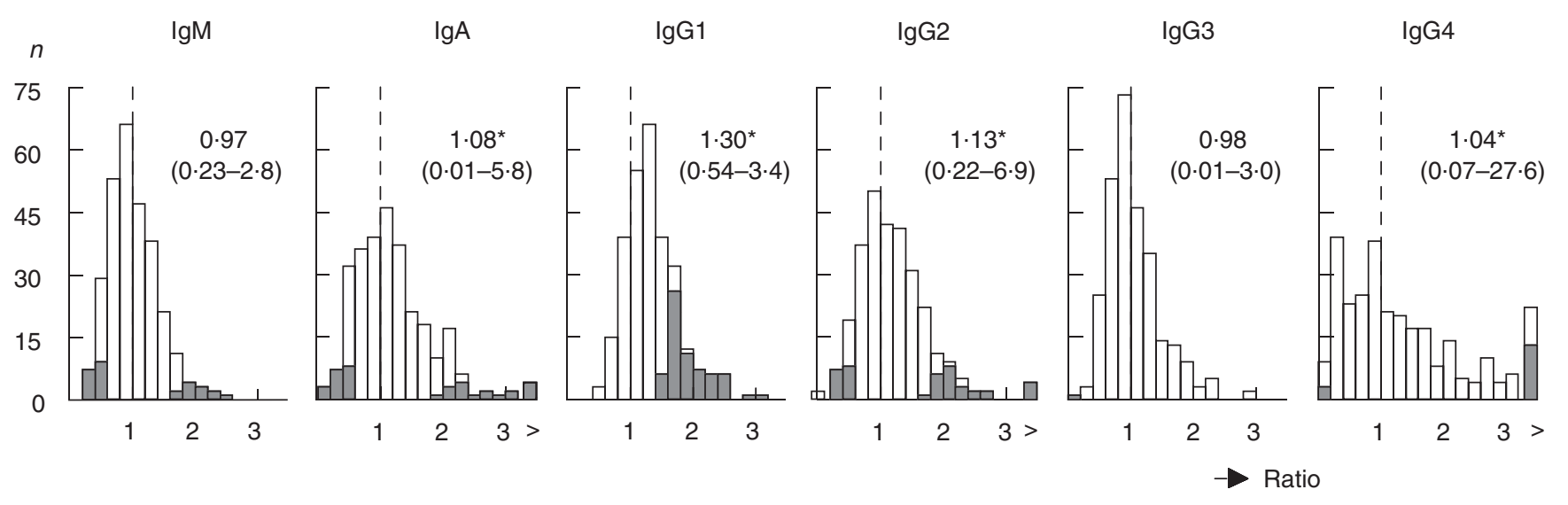

Fig. 1. Ratio between the observed immunoglobulin (Ig) (sub)class concentrations in children from whom a blood sample was obtained at intake $(n=282)$ and the published median of normal levels [24,25]. IgA, IgG1, IgG2 and IgG4 concentrations were significantly higher in our cohort than the reported median Ig (sub)class concentrations of healthy children of the corresponding ages $(* P<0 \cdot 001)$. Grey areas represent children with Ig (sub)class concentrations below the lower limit or above the upper limit of the normal range. The median ratio of all $\mathrm{Ig}$ (sub)classes as well as the range (between brackets) are given. >: ratio $\geq 3 \cdot 4$. 
(sub)classes with one exception. Children with an IgG1 concentration above the upper limit of the normal range were significantly younger than children with an $\mathrm{IgG} 1$ concentration within the normal range (median age at intake 4.5 and 6.9 years, respectively, $P<0 \cdot 01)$.

At the time of the first blood sampling the seizure frequency was known in 163 of the 282 patients. Ig levels did not differ significantly between children who had experienced a single seizure and those who had multiple seizures. However, there was a significant negative correlation between the overall seizure frequency and the IgG1 level corrected for age $(P=0 \cdot 003)$, indicating that IgG1 was lower in children with higher seizure frequency. For the other Ig (sub)classes no significant correlations were found. No significant differences in Ig were observed between the different epilepsy syndromes as classified by the ILAE (including six children with West syndrome and two with Lennox-Gastaut syndrome) [21].

In our control group of 76 healthy children, who were bone marrow transplant donors, none of the median concentrations of the Ig classes and IgG subclasses were significantly different from those reported in healthy children of the corresponding age group. On an individual basis, abnormal concentrations of one or more of the Ig classes and IgG subclasses were detected only in sporadic cases, and only $5 \%$ of the children in our control group showed an increase of IgG1 (data not shown).
Immunoglobulin levels after using anti-epileptic drugs for 9-18 months

In $127(45 \%)$ of the 282 children, a second blood sample was obtained after 9-18 months of continuous AEDs use. Median age at onset of epilepsy, sex, aetiology, distribution of epilepsy syndromes and median Ig ratios at intake did not differ significantly between these children and those from whom no second blood sample was taken.

After using AEDs for 9-18 months, $\operatorname{IgA}(P<0 \cdot 001)$ and $\operatorname{IgG} 4$ $(P<0 \cdot 01)$ levels had decreased significantly compared to the levels at intake; IgG1 $(P<0 \cdot 001)$ and IgG3 $(P<0 \cdot 01)$ levels had significantly increased (Fig. 2). The significant decrease in $\operatorname{IgA}$ and $\mathrm{IgG} 4$ levels led to normalization of these levels with a median ratio of approximately 1 ; there was no difference in the number of children with $\operatorname{IgA}$ or IgG4 concentrations below the lower limit of the normal range at intake and after AEDs use for 9-18 months. No new cases with an absolute IgA deficiency were observed. The significant increase of IgG1 after 9-18 months of AEDs use, however, led to $38 \%$ of the 127 children having an IgG1 concentration above the upper limit of the normal range (Fig. 2), which was significantly more than before treatment $(24 \%, P<0 \cdot 01)$. The change in IgG1 levels over time did not differ significantly between different aetiologies or different outcomes. Children with generalized epilepsy, however, showed a significant increase of the IgG1 level $(P<0 \cdot 001)$ while the

$\lg \mathrm{M}$

$\lg A$

$\operatorname{lgG1}$

$\lg \mathrm{G} 2$

$\lg G 3$

$\lg G 4$
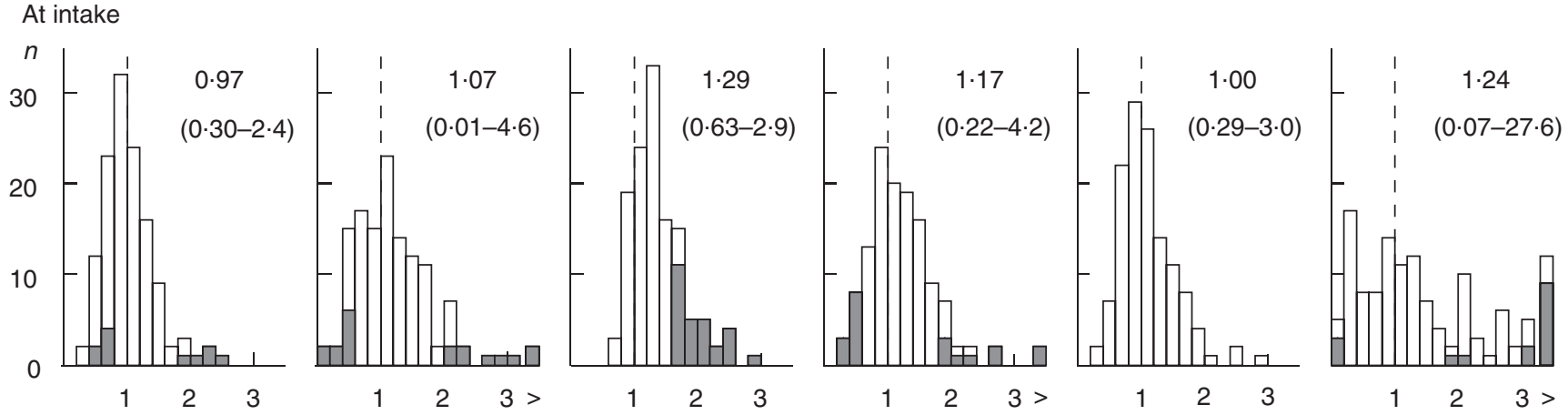

After 9-18 months
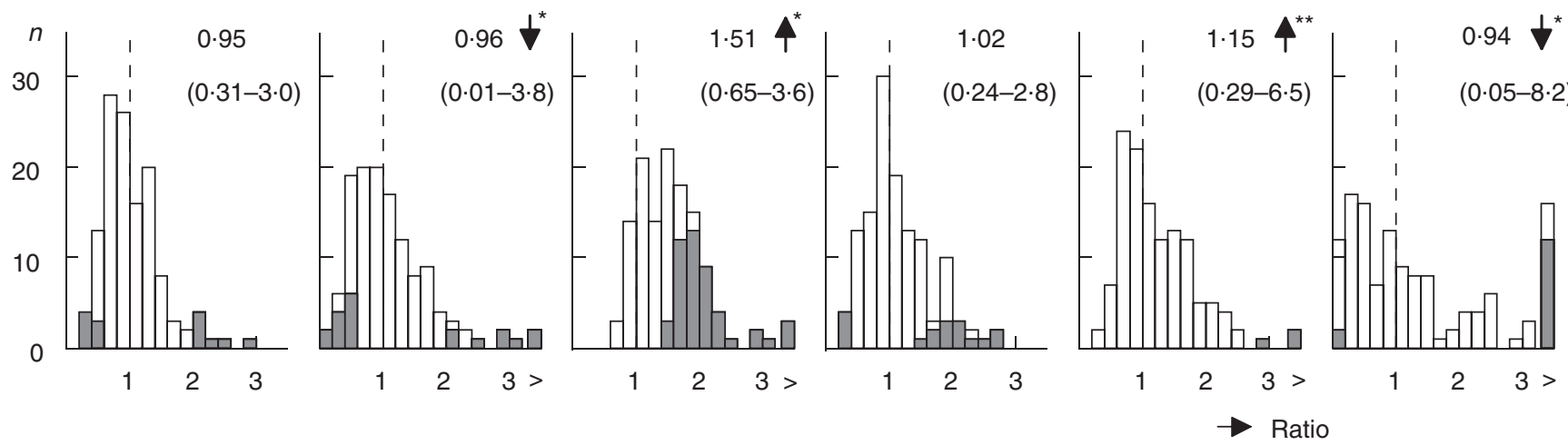

Fig. 2. Ratio between the observed immunoglobulin ( $\mathrm{Ig})$ (sub)class concentrations in children $(n=127)$ from whom a blood sample was obtained at intake (upper panel) and after 9-18 months of AEDs use (lower panel), and the reported median of normal levels [24,25]. IgA (* $P<0 \cdot 001$ and IgG4 $(* * P<0 \cdot 01)$ levels had decreased significantly as compared to the levels at intake; IgG1 $(* P<0 \cdot 001)$ and IgG3 (**P<0.01) levels showed a significant increase. Grey areas represent children with Ig (sub)class concentrations below the lower limit or above the upper limit of the normal range. The median ratio of all Ig (sub)classes as well as the range (between brackets) are given. >: ratio $\geq 3 \cdot 4$. 
median IgG1 level remained approximately the same in children with localization-related epilepsy. Furthermore, aetiology, outcome and epilepsy syndrome distribution were not significantly different between children with an IgG1 concentration above the upper limit of the normal range and children with an IgG1 concentration within the normal range. The age at the time of the second blood sample was, however, significantly lower in children with an IgG1 concentration above the upper limit of the normal range than in children with an $\mathrm{IgG} 1$ concentration within the normal range (median age 4.3 and 9.5 years, respectively, $P<0.001$ ). The increase of IgG3 led to $2 \%$ of the children having an IgG3 concentration above the upper limit of the normal range after 918 months of AEDs use (Fig. 2).

\section{Anti-epileptic drugs}

To determine more closely the influence of distinct anti-epileptic drugs, changes of Ig concentrations in children that used carbamazepine or valproic acid as monotherapy were investigated. After 9-18 months, 34 (27\%) children had continuously received carbamazepine monotherapy and $60(47 \%)$ valproic acid monotherapy. Of the other 33 children, seven used ethosuximide, two vigabatrin, one phenytoin and 23 had polytherapy. Because of the small size of the groups, the influence of these anti-epileptic drugs on the immunoglobulins could not be determined.

Of the 34 children using carbamazepine, seven (21\%) suffered from epilepsy of idiopathic aetiology, 13 (38\%) from epilepsy of cryptogenic aetiology and $14(41 \%)$ from epilepsy of remote symptomatic aetiology. Thirty-one ( $91 \%$ ) had localization-related epilepsy and three $(9 \%)$ generalized epilepsy. In children on carbamazepine monotherapy, IgA $(P<0 \cdot 01)$ and $\operatorname{IgG} 4(P<0 \cdot 01)$ levels had decreased significantly over time, leading to normalization of the median IgA and IgG4 levels (Fig. 3a).

Of the 60 children using valproic acid, 44 (73\%) suffered from epilepsy of idiopathic aetiology, three (5\%) from epilepsy of cryptogenic aetiology and 13 (22\%) from epilepsy of remote symptomatic aetiology. Seven (12\%) had localization-related epilepsy and $52(87 \%)$ generalized epilepsy. In children on valproic acid monotherapy, IgA levels showed a significant decrease to normal concentrations with a median ratio of approximately $1(P<0 \cdot 01)$ and a significant increase of IgG1 levels $(P<0 \cdot 001$, Fig. 3b). After 9-18 months of treatment, $26(43 \%)$ of these children had an IgG1 above the upper limit of the normal range compared to 18 $(30 \%)$ at intake. This difference was not statistically significant. To investigate whether the observed increase in $\mathrm{IgG1}$ was due merely to the use of valproic acid, it was tested whether IgG1 levels decreased after discontinuation of valproic acid. Blood samples were taken from 10 children who were excluded from the analysis described above because they discontinued AEDs within 9 months, just before discontinuation of valproic acid and 4-11 months after discontinuation. IgG1 levels significantly decreased after discontinuation of valproic acid $(P<0.05$, data not shown). Furthermore, we investigated whether IgG1 levels differed between children that had polytherapy with $(n=18)$ or without $(n=5)$ valproic acid. These groups did not differ significantly in age, aetiology, epilepsy syndrome distribution, outcome and IgG1 levels at intake. After using therapy for 9-18 months, however, IgG1 levels were significantly higher in the group that used polytherapy with valproic acid than in the group without valproic acid $(P<0 \cdot 05$, data not shown).

When comparing Ig levels between children using carbamazepine and children using valproic acid, it was observed that IgM levels were significantly lower in the group using carbamazepine than in the group using valproic acid $(P<0 \cdot 01)$. Furthermore, IgG1 levels were significantly higher in the group using valproic acid than in the group using carbamazepine $(P<0 \cdot 01)$. The other Ig levels did not differ significantly between the two groups.

\section{DISCUSSION}

This study focuses on a large cohort of children with one or more unprovoked epileptic seizures followed prospectively from their first visit to a specialist clinic. Serum samples were already available early after the first presentation with seizures and before the initiation of any treatment with AEDs. In addition, a second serum sample was available in about half of the patients at followup after 9-18 months of AEDs use.

Since median concentrations of the Ig (sub)classes in serum samples of our control group of 76 healthy children were not significantly different from the reported median values in healthy age-matched controls [24,25], our experimental approach can be considered to be valid. Therefore, the observed increase in the median serum concentration of $\mathrm{IgA}, \mathrm{IgG} 1, \mathrm{IgG} 2$ and IgG4 (corrected for age) in our cohort of children with newly diagnosed epilepsy indicates that disturbances of humoral immunity are already present before the initiation of medical treatment, and that these disturbances are independent from aetiology and the type of epilepsy syndrome. However, increased IgG1 concentrations were especially detected in young children. It has to be realized that the children from whom no blood sample was taken at intake were significantly younger than the children from whom this blood sample was obtained. Therefore, the influence of epilepsy on IgG1 might be underestimated because of a sampling bias.

To our knowledge, this is the first study in which only children are enrolled and in which serum Ig concentrations were already measured within a short period (median: 69 days) after the first presentation with epileptic seizures and before the start of AEDs. Some other reports have also described alterations of humoral immunity in epilepsy patients who were not on AEDs $[13,15]$, whereas Lenti et al. did not observe alterations in untreated patients [26]. Unfortunately, the time period passed between the first presentation of seizures and the measurement of serum Ig concentrations was not indicated in these papers.

The underlying mechanism for the observed disturbances of humoral immunity in children at an early stage of epilepsy remains unknown. One can speculate that infections might have triggered the immune response, as suggested by the increased serum concentrations of various IgG subclasses and IgA, and concomitantly might have caused a disturbance of the blood-brain barrier. Exposure of formerly hidden antigens of the CNS to the immune system might lead consequently to seizures possibly mediated by cross-reactive autoantibodies produced in response to the infectious agents or by antibodies induced by antigens of the CNS itself. However, in our study cohort there is no formal indication for such an order of events. At intake, each of the 282 children showed normal leucocyte counts and a normal leucocyte differentiation, suggesting that infections in this population at that time point were very rare. On the other hand, $5 \cdot 3 \%$ of the children in our study cohort showed an increased serum concentration of $\operatorname{IgD}$ at intake, in comparison with none of the children in the control group (data not shown). At the Department of Paediatrics of the Leiden University Medical Centre, elevated levels of $\operatorname{IgD}$ are particularly detected in children suffering from 


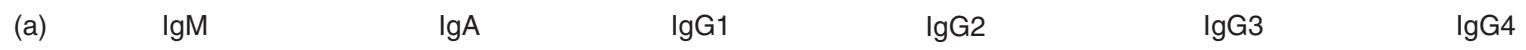

Carbamazepine group at intake
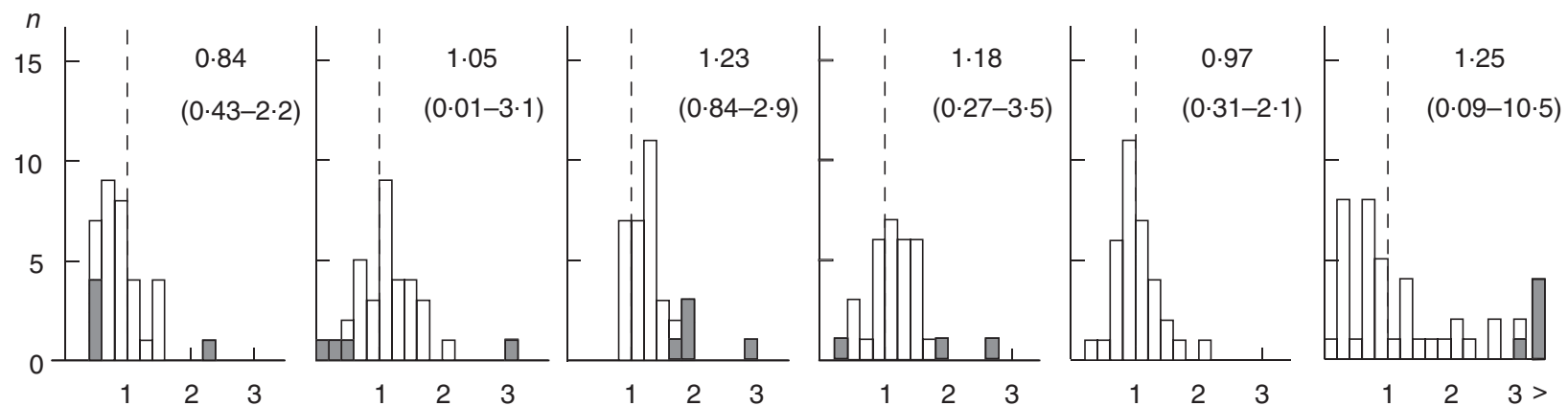

Carbamazepine group after 9-18 months

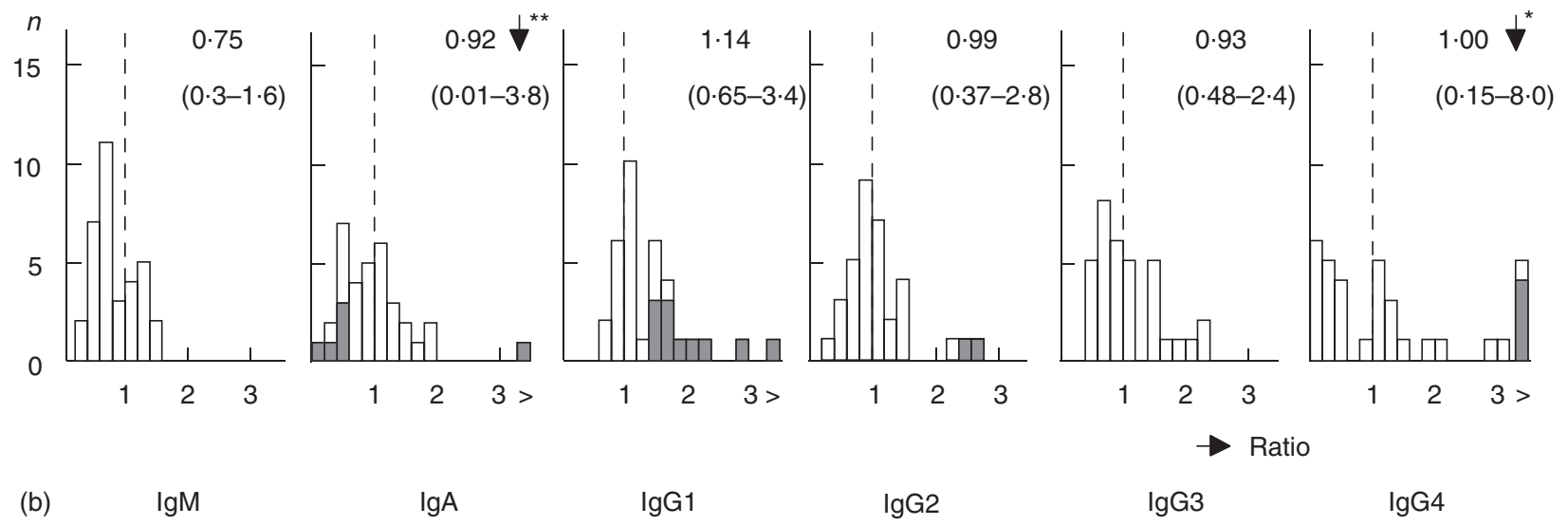

Valproic acid group at intake
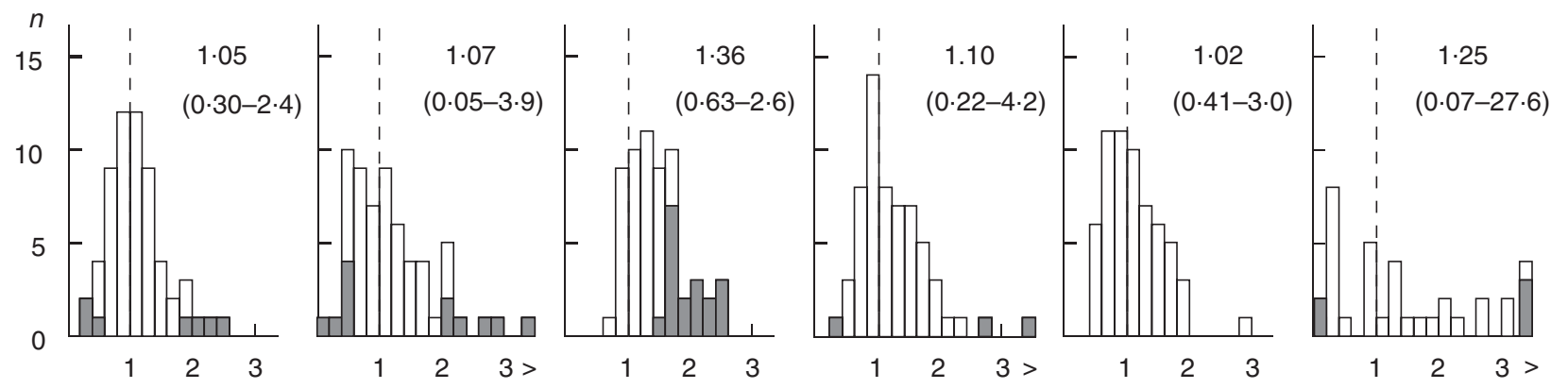

Valproic acid group after $9-18$ months
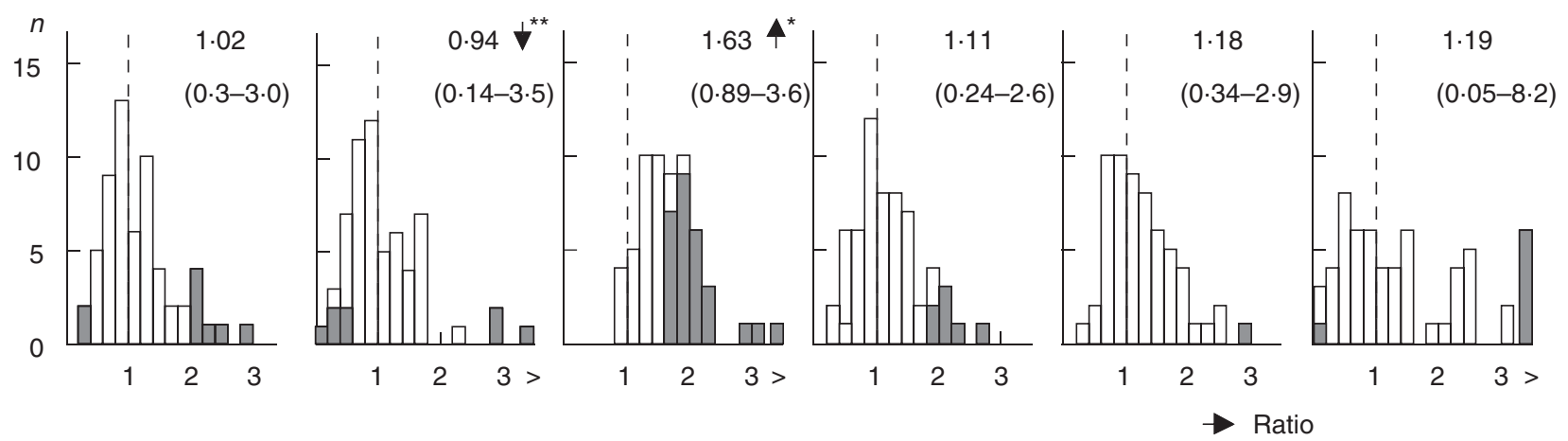

Fig. 3. Ratio between the observed immunoglobulin (Ig) (sub)class concentrations at intake (upper panel) and after 9-18 months (lower panel) of (a) carbamazepine $(n=34)$ or (b) valproic acid $(n=60)$ monotherapy, and the reported normal levels [24,25]. In children using carbamazepine, IgA and IgG4 levels showed a significant decrease $(* * P<0 \cdot 01)$. The children that used valproic acid had a significant decrease of IgA levels $(* * P<0 \cdot 01)$ and a significant increase of IgG1 levels $(* P<0 \cdot 001)$. Grey areas represent children with Ig (sub)class concentrations below the lower limit or above the upper limit of the normal range. The median ratio of all Ig (sub)classes as well as the range (between brackets) are given. $>$ : ratio $\geq 3 \cdot 4$. 
recurrent respiratory tract infections or autoimmune diseases (e.g. juvenile idiopathic arthritis). We did not find a correlation between an increase of $\mathrm{IgG1}$ and an elevation of $\mathrm{IgD}$ in our cohort. Because no clinical information about the presence of, e.g. respiratory infections was obtained at intake, no statement can be made about the relation between infections and epilepsy in our cohort. As a first step to investigate the possible aetiology of the various forms of childhood epilepsy more closely, it will be relevant to measure antibody titres in these sera against bacterial and viral micro organisms and against a panel of autoantigens.

The lack of an association between the occurrence of abnormally low or high concentrations of serum Ig classes or IgG subclasses early in the disease and the outcome (terminal remission) of the epilepsy measured 5 years after intake, indicates that Ig levels before the start of AEDs treatment are not useful as markers for the early recognition of patients with intractable epilepsy. Ig concentrations at the time of the second blood sampling were also not significantly different in children with different outcomes.

The decrease of IgA and IgG4 and increase of IgG1 and IgG3 after 9-18 months of AEDs treatment in the whole study population of 127 children can, at least partially, probably be attributed to the effect of medication. In children on carbamazepine monotherapy for 9-18 months, IgA and IgG4 concentrations showed a decrease in comparison with the level before the initiation of treatment. Regarding the decrease of $\operatorname{IgA}$, our observation is in line with an earlier report concerning adults on carbamazepine [10]. However, in our study cohort we did not find a significant decrease of IgM as reported previously by others in adults [10] and children [13]. Furthermore, our data are contradictory to other reports in adults [12] and children [26], which showed no effect of carbamazepine treatment on serum concentrations of any of the major Ig isotypes. In a combined population of adults and children, Gilhus and Lea have described an association between carbamazepine monotherapy and a decrease of $\mathrm{IgG} 2$, while IgG4 remained unaffected [11]. Interpretation of the divergence of these observations from our results is impossible, due to the lack of data obtained before the start of treatment in most of the studies, the variable composition of the study population regarding aetiology and type of epilepsy syndromes, the usually small size of the study groups and the variable duration of medication use ranging from months to over 10 years. Moreover, information on the start of medication related to the onset of epilepsy is not available from most of the published studies.

Our results indicate that valproic acid, as monotherapy or as part of a combined therapy, is associated with an increase of IgG1. In addition, in children on monotherapy with valproic acid, IgA decreased to normal levels. Data in the literature on the effect of valproic acid on the concentration of serum Ig classes are scarce and even lacking when IgG subclasses are concerned [14$16,26,27]$. Although the same constraints hold for these studies as indicated above for the studies on carbamazepine, the common observation arose in these studies that the concentrations of the major Ig isotypes in serum are unaffected in patients treated with valproic acid. Unfortunately, we have no information about the change in serum immunoglobulin concentrations over time in non-treated patients with epilepsy. Therefore, we cannot exclude that the observed changes are caused predominantly by the epilepsy itself and not by the use of AEDs. We observed, however, that IgG1 levels decreased significantly after withdrawal of valproic acid, leading to the conclusion that valproic acid was indeed responsible for the increase of $\operatorname{IgG} 1$.
It is attractive to speculate that the divergent development of Ig (sub)class concentration in children treated with carbamazepine and valproic acid, respectively, might be associated with a different effect of these AEDs on B cell maturation (i.e. Ig isotype switching), either directly at the B cell level or at the level of regulatory T lymphocytes.

In conclusion, humoral immunity is already altered in children early after the first presentation with epileptic seizures. Whether this is the consequence of an exogenous event, such as the occurrence of infection, and to what extent this is related to an interaction of the CNS and the immune system remains to be evaluated. Treatment with carbamazepine and valproic acid is associated with significant changes of serum concentrations of distinct $\mathrm{Ig}$ isotypes and $\mathrm{IgG}$ subclasses. To outline the clinical relevance of altered immunity in children with epilepsy, further research has to be performed in which the medical history of each child regarding infections is recorded from intake onwards and in which serum Ig concentrations of non-treated children are compared over time with those of children using AEDs.

\section{ACKNOWLEDGEMENTS}

The Dutch Study of Epilepsy in Childhood was supported financially by the National Epilepsy Fund, Houten, the Netherlands (A72 and A85). P. M. C. Callenbach is a research fellow supported by a grant from the Netherlands Organization for Health, Research and Development (ZonMw, 940-33-030) and the National Epilepsy Fund (98-14).

\section{REFERENCES}

1 Palace J, Lang B. Epilepsy: an autoimmune disease? J Neurol Neurosurg Psychiatry 2000; 69:711-4.

2 Aarli JA. Epilepsy and the immune system. Arch Neurol 2000; 57:1689-92.

3 Rogers SW, Andrews PI, Gahring LC et al. Autoantibodies to glutamate receptor GluR3 in Rasmussen's encephalitis. Science 1994; 265:648-51.

4 van Engelen BG, Renier WO, Weemaes CM, Gabreels FJ, Meinardi H. Immunoglobulin treatment in epilepsy, a review of the literature. Epilepsy Res 1994; 19:181-90.

5 Duse M, Notarangelo LD, Tiberti S, Menegati E, Plebani A, Ugazio AG. Intravenous immune globulin in the treatment of intractable childhood epilepsy. Clin Exp Immunol 1996; 104 (Suppl. 1):71-6.

6 Seager J, Jamison DL, Wilson J, Hayward AR, Soothill JF. IgA deficiency, epilepsy, and phenytoin treatment. Lancet $1975 ; \mathbf{2}: 632-5$.

7 Aarli JA. Changes in serum immunoglobulin levels during phenytoin treatment of epilepsy. Acta Neurol Scand 1976; 54:423-30.

8 Gilhus NE, Aarli JA. The reversibility of phenytoin-induced IgA deficiency. J Neurol 1981; 226:53-61.

9 Gilhus NE, Lea T. IgG subclasses in epileptic patients treated with phenytoin. J Neurol 1989; 236:149-52.

10 Gilhus NE, Strandjord RE, Aarli JA. The effect of carbamazepine on serum immunoglobulin concentrations. Acta Neurol Scand 1982; 66:172-9.

11 Gilhus NE, Lea T. Carbamazepine: effect on IgG subclasses in epileptic patients. Epilepsia 1988; 29:317-20.

12 Pacifici R, Paris L, Di Carlo S, Pichini S, Zuccaro P. Immunologic aspects of carbamazepine treatment in epileptic patients. Epilepsia 1991; 32:122-7.

13 Basaran N, Hincal F, Kansu E, Ciger A. Humoral and cellular immune parameters in untreated and phenytoin- or carbamazepine-treated epileptic patients. Int J Immunopharmacol 1994; 16:1071-7. 
14 Garzon P, Gonzalez-Cornejo S, Roman-Maldonado S, Navarro-Ruiz A. Valproic acid and phenytoin effects on serum proteins and immunoglobulins of epileptic patients. General Pharmacol 1985; 16:411-3.

15 Bostantjopoulou S, Hatzizisi O, Argyropoulou O et al. Immunological parameters in patients with epilepsy. Funct Neurol 1994; 9:11-5.

16 Hemingway C, Leary M, Riordan G, Schlegal B, Walker K. The effect of carbamazepine and sodium valproate on the blood and serum values of children from a third-world environment. J Child Neurol 1999; 14:751-3.

17 Peters ACB, Brouwer OF, Geerts AT, Arts WFM, Stroink H, van Donselaar CA. Randomized prospective study of early discontinuation of antiepileptic drugs in children with epilepsy. Neurology 1998; 50:724-30.

18 Stroink H, Brouwer OF, Arts WFM, Geerts AT, Peters ACB, van Donselaar CA. The first unprovoked, untreated seizure in childhood: a hospital based study of the accuracy of the diagnosis, rate of recurrence, and long term outcome after recurrence. The Dutch Study of Epilepsy in Childhood. J Neurol Neurosurg Psychiatry 1998; 64:595-600.

19 Arts WFM, Geerts AT, Brouwer OF, Peters ACB, Stroink H, van Donselaar CA. The early prognosis of epilepsy in childhood: the prediction of a poor outcome. The Dutch Study of Epilepsy in Childhood. Epilepsia 1999; 40:726-34

20 Commission on Classification and Terminology of the International
League Against Epilepsy. Proposal for revised clinical and electroencephalographic classification of epileptic seizures. Epilepsia 1981; 22:489-501.

21 Commission on Classification and Terminology of the International League Against Epilepsy. Proposal for revised classification of epilepsies and epileptic syndromes. Epilepsia 1989; 30:389-99.

22 Commission on Epidemiology and Prognosis ILAE. Guidelines for epidemiologic studies on epilepsy. Epilepsia 1993; 34:592-6.

23 Jol-Van der Zijde CM, Labadie J, Vlug A, Radl J, Vossen JM, Van Tol MJ. Dot-immunobinding assay as an accurate and versatile technique for the quantification of human IgG subclasses. J Immunol Meth 1988; 108:195-203.

24 Cejka J, Mood DW, Kim CS. Immunoglobulin concentrations in sera of normal children: quantitation against an international reference preparation. Clin Chem 1974; 20:656-9.

25 Central Laboratory of the Netherlands Red Cross Blood Transfusion Service (CLB). Vademecum Diagnostic Research: Amsterdam, The Netherlands 1997.

26 Lenti C, Masserini C, Peruzzi C, Guareschi CA. Effects of carbamazepine and valproate on immunological assessment in young epileptic patients. Ital J Neurol Sci 1991; 12:87-91.

27 De Sarro GB, Berlinghieri MC, Elia M et al. Does antiepileptic therapy affect immune response? J Chemother 1998; 10:184-6. 